\title{
LOCATION OF THE ADVENTITIOUS FACTORS IN HAEMOLYTIC DISEASE OF THE NEWBORN
}

\author{
BY \\ GEORGE KNOX, SHEILAGH MURRAY, AND WILLIAM WALKER \\ From the Department of Child Health, University of Durham at Kings College, and \\ the Regional Blood Transfusion Centre, Newcastle-upon-Tyne
}

It is by now well established that the frequencies of $\mathrm{ABO}$ mating types among parents of children with $\mathrm{Rh}$ haemolytic disease of the newborn are not the same as in the general population. There is a reduced frequency of matings in which the mother has antibodies to ABO-antigens possessed by her husband. It is possible that incompatible foetal blood escaping into the mother's circulation is so rapidly destroyed that its ability either to sensitize or later to immunize the mother against $R h$ substance is impaired. Nevanlinna and Vainio (1956) have also shown that within affected families the proportion of healthy children who are incompatible with the mother for the $\mathrm{ABO}$ groups is even lower than the proportion of affected ones. They have argued that because, on average, healthy children occur earlier in these families than do affected ones, the protective effect of ABO-incompatibility is chiefly exercised at the stage of sensitization (sensibilization in their terms) rather than during the pregnancy when antibodies are first produced in sufficient quantities to cause disease.

Knox and Walker (1957) constructed an argument based upon the birth rank distribution of affected infants which led to a conclusion in the form of two alternatives. One was complex, requiring an extra genetic determinant, together with a risk of sensitization of the order of 0.39 at each Rh-positive pregnancy, and a similar risk of immunization at each subsequent Rh-positive pregnancy. The other was much simpler (and therefore preferred), requiring no important extra genetic determinants and a single important adventitious factor related to only one of the pregnancies, with a risk of occurrence of 1/17. The method could not distinguish which pregnancy this was.
It has since occurred to us that the protection conferred by ABO-incompatibility, apart from its intrinsic interest, may also be used as a tool to extend our previous argument. For if it can be shown precisely at which pregnancy or pregnancies the protection is conferred, then that also must be the location of an important adventitious factor against which the incompatibility protects.

It is therefore necessary to press the arguments of Nevanlinna and Vainio to a more precise and critical stage; to deduce in detail the expected consequences of the hypothesis they have set up and of alternative hypotheses; to examine the facts in relationship to these deductions. This is the purpose of the present paper.

\section{Hypothetical Deductions}

The simplest situation in which we can examine the consequences of different plausible mechanisms of $\mathrm{Rh}$-sensitization and immunization is in families in which the first child is healthy and the second child affected. Obviously, if ABO-incompatibility were irrelevant to the situation, we should expect both first and second children to be incompatible with their mothers in the same proportions as in the population. If incompatibility were relevant in the way Nevanlinna and Vainio suggest, the proportion of incompatible infants should be reduced especially in the first pregnancy, with a limiting value of zero. The proportion in the second pregnancy should also be reduced because of selection of particular parental mating types, but not as low as in the first pregnancy. On the other hand, if ABO-incompatibility exerted a protective effect only in the second pregnancy, these relationships would be reversed with an especially 
low frequency of incompatibility in the second pregnancy and a less marked reduction in the first.

Unfortunately, this does not exhaust the possibilities. It may be that incompatibility operates in both situations, in which case we might expect a more equal reduction of frequency in the two situations.

It has not been possible to derive the expected frequencies of $\mathrm{ABO}$-incompatible foetuses for all the mechanisms, but it has been possible to derive those for the limiting cases. These derivations are now presented.

The $\mathrm{ABO}$ mating types (ignoring the subdivisions of A) may be put into three classes. These classes may be designated $1, \frac{1}{2}, 0$, according to the respective probabilities of any particular child being ABOcompatible. The frequencies of individual matings are derived from the products of the parental genotype frequencies (as given by Race and Sanger, 1958), doubling of products when the parents have different genotypes being unnecessary here because we have taken account of maternal and paternal identities.

The summed frequencies of the three classes show that 5.85 per cent. of all matings can never produce an ABO-compatible foetus, while 28.06 per cent. will do so in half of their children. The remaining 66.09 per cent. will always have compatible children. Furthermore, it is simply derived that $80 \cdot 12$ per cent. of all foetuses in the population are compatible with their mothers. This gives the expectation for the null hypothesis; if the ABO groups were irrelevant to the formation of Rhesus antibodies we should expect 19.88 per cent. both of first babies and of second babies in the specified families to be incompatible with their mothers.

The hypothesis suggested by Nevanlinna and Vainio (1956) is that incompatibility at the first of the two pregnancies is protective. In the limiting situation of complete protection we expect the frequency of incompatibility in this pregnancy, in families selected through an affected second child, to be zero. In this event the parental mating types would consist of the following relative proportions:

$\begin{array}{ccccr}\text { Type } 1 & \ldots & \ldots & \ldots & 66.09 \times 1 \\ \text { Type } \frac{1}{2} & \ldots & \ldots & \ldots & 28.06 \times \frac{1}{2} \\ \text { Type 0 } & \ldots & \ldots & \ldots & 5.85 \times 0 .\end{array}$

This gives absolute proportions of 82.49 per cent. Type 1 and $17 \cdot 51$ per cent. Type $\frac{1}{2}$. The second babies consequently will be incompatible and compatible in the proportions $8 \cdot 76$ per cent. and $91 \cdot 24$ per cent.
The antithesis of this hypothesis is that ABO-incompatibility protects only at the second pregnancy. In this case, the reasoning is a mirror image of that already presented, the expected proportions of incompatible foetuses being zero in the second pregnancy, 8.76 per cent. in the first.

The hypothesis that complete protection occurs in both pregnancies clearly results in zero incompatibility in each situation. Heterogeneity, in the sense that some families are protected in one manner, others in another, others not at all, will result in proportions which are mixtures of the results for individual hypothetical mechanisms.

The four main hypotheses, the limits of a variety of possible ones, are presented in the Table.

\section{TABLE}

EXPECTED PROPORTIONS (PER CENT.) OF ABO-INCOMPATIBLE FOETUSES IN FIRST AND SECOND PREGNANCIES, WHERE THE SECOND CHILD HAS HAEMOLYTIC DISEASE OF THE NEWBORN

\begin{tabular}{|c|c|c|}
\hline Hypothesis & $\begin{array}{c}\text { First } \\
\text { Children }\end{array}$ & $\begin{array}{c}\text { Second } \\
\text { Children }\end{array}$ \\
\hline No protection from incompatibility & $19 \cdot 9$ & $19 \cdot 9$ \\
\hline Full protection at first pregnancy only & 0 & $8 \cdot 8$ \\
\hline Full protection at second pregnancy only & $8 \cdot 8$ & 0 \\
\hline $\begin{array}{c}\text { Full protection when either pregnancy is } \\
\text { incompatible } \quad \ldots \\
.\end{array}$ & 0 & 0 \\
\hline
\end{tabular}

\section{ObServations}

(i) Almost all infants with haemolytic disease of the newborn in the Newcastle area are treated in this Department, and in the period 1948 to 1958 we have records of 331 babies who were the second baby in their families and the first affected, and for whom we knew the ABO groups of both the baby and its mother. Of these 331, thirty $(9.06 \pm 1.58$ per cent.) were incompatible.

(ii) We knew the $\mathrm{ABO}$ group of the first child also in some of these families, but since our data were not complete we could not be certain that selection in terms of some relevant variable had not taken place. We wrote, therefore, and asked all those mothers who lived within a reasonable distance and whose record was incomplete, if they would attend with the older child. In this way we collected a sample of data which we suppose not to be substantially biased, although some failed to attend.

Of 71 children in this sample, three $(4 \cdot 2$ per cent.) were incompatible.

By adding first children whose blood groups were known previously and who lived outside the area of 
the supplementary survey, we have four of 99 (4 per cent.) incompatible. Since the addition has not materially altered the result it is probably valid to use all the information.

\section{Discussion}

The proportion of incompatibles is significantly less than in the general population both at the first and at the second pregnancy, confirming that protection occurs. The proportion is lower at the first pregnancy than at the second, suggesting that protection occurs especially at the stage of sensitization, but the difference is not statistically significant and the protection is obviously not complete.

However, with four of 99 first children incompatible, we can say that the proportion is less than the minimum expected for full protection at the second pregnancy to a degree which approaches statistical significance $\left(\chi_{(1)}^{2}=2 \cdot 87, \mathrm{P}<0 \cdot 1, \ldots\right.$ or $\mathbf{P}<0.05$ on a single tail test), and that it is quite certainly less than the proportion expected for any degree of protection at the second pregnancy which would be consistent with an observed incompatibility rate there of 9.06 per cent.

On the other hand, the degree of protection necessary at the first pregnancy in order to produce an incompatibility rate of 9.06 per cent. among affected second babies is consistent with the findings at the first pregnancy. It is true that the proportion of incompatibles at the first pregnancy is somewhat higher than might have been expected having regard to the proportion at the second and that it is possible that there is some protection at the immunization stage also. But this cannot be great, and most if not all of the protection appears to be conferred at the stage of sensitization.

The proportion of incompatibles at the first pregnancy suggests that an $\mathrm{ABO}$-incompatible $\mathrm{Rh}$-positive foetus carries about 20 per cent. of the normal risk of sensitizing an Rh-negative mother. This corresponds with the estimate reached by Nevanlinna and Vainio (1956). The proportion of incompatibles at the second pregnancy suggests that the risk is somewhat smaller and seems to indicate that 20 per cent. must be near the upper limit.
For the purposes of our present thesis and the extension of our previous argument, it is to be concluded that important unknown adventitious factors, which determine that one woman becomes immunized while another does not, are chiefly concentrated at the sensitization stage, and that when she embarks upon the next $\mathrm{Rh}$-positive pregnancy the outcome in terms of immunization is already decided.

\section{SUMMARY}

Previous work has suggested that ABO-incompatibility of husband and wife may protect against Rhesus immunization of the wife. It has also been shown that, besides this incompatibility effect, imimportant unknown adventitious factors are at work to determine whether immunization occurs. It is argued that the timing of the protective effect, which can be inferred, can be used to identify the location of the adventitious factors. It is shown that $\mathrm{ABO}$ protection occurs at the stage of sensitization and inferred that the adventitious factors must operate here also.

\section{REFERENCES}

Knox, G., and Walker, W. (1957). Brit. J. prev. soc. Med., 11, 126. Nevanlinna, H. R., and Vainio. T. (1956). Vox Sang. (Basel), n.s.1, 26. Race, R. R., and Sanger, R. (1958). "Blood Groups in Man", 3rd ed. Blackweli, Oxford.

\section{ADDENDUM}

Since submitting this paper we have re-examined the four families where the first child was ABOincompatible. The $\mathrm{ABO}$ and $\mathrm{Rh}$ groups were all confirmed, but one case was found not to be a case of $\mathrm{Rh}$ isoimmunization. The diagnosis had been accepted originally upon a doubtful result since a request for further blood samples had not been met; the error was due to cold agglutinins. This strengthens our conclusions, but we have not re-analysed our data since this would not be legitimate without serological re-examination of all cases.

Another of the four children was stillborn, but we examined the ABO secretor status of the other two. One was a secretor and the other was not. There is no evidence here that the secretor status might explain the occurrence of exceptional sensitizations in the presence of incompatibility. 